\title{
Factors associated with discrepancies between poor dyspnea perception and abnormal lung function in 65 asthmatic patients
}

\author{
Asmaa Jniene ${ }^{1,2}$, Leila Achachi ${ }^{1}$, Mustapha El Bakkali², Laila Herrak ${ }^{1}$, Aziza Rhanim¹, \\ Souad Aboudrar ${ }^{2}$, Taoufiq Dakka ${ }^{2}$, Mustapha El Ftouh ${ }^{1}$
}

1. Department of Pulmonology, Ibn Sina Hospital, Ibn Sina University Hospital Center, Faculty of Medicine and Pharmacy, Mohammed V University, Rabat, Morocco

2. Exercise Physiology and Autonomic Nervous System Team "EPE-SNA", Laboratory of Physiology, Faculty of Medicine and Pharmacy, Mohammed V University, Rabat, Morocco

\section{Emails:}

Leila Achachi: achachi_leila@yahoo.fr; Mustapha El Bakkali: elbakkali@hotmail.fr; Laila Herrak: herraklaila@yahoo.fr; Aziza Rhanim: aziza.rhanim@gmail.com; Souad Aboudrar: souadaboudrar@yahoo.fr; Taoufiq Dakka: t.dakka@um5s.net.ma; Mustapha El Ftouh: ftoh18@yahoo.fr

\begin{abstract}
Background: Dyspnea is a subjective symptom of asthma whose perception is characterized by an interindividual variability. Poor dyspnea perception is usually associated with increased risk of exacerbation and may lead to inappropriate asthma management and under-treatment. We sought to identify factors associated with discrepancies between poor dyspnea perception and abnormal lung function in patients with moderate persistent asthma.

Methodology: 65 patients, who attended their scheduled follow-up pulmonology consultation at Ibn Sina Hospital (Rabat - Morocco), underwent interrogation including modified Medical Research Council (mMRC) scale for dyspnea, physical examination and spirometry. Two groups, those with $\mathrm{mMRC}<2$ (poor dyspnea perceivers) and those with mMRC scale $\geq$ 2 were compared.

Results: Poor dyspnea perception was found in 21 patients (32.3\%). Associated factors were male sex (p : 0.03), low socio-economic income ( $p: 0.01)$, an onset of symptoms greater than 10 years ( $\mathrm{p}: 0.01)$, BMI $\geq 25 \mathrm{Kg} / \mathrm{m} 2$ ( $\mathrm{p}: 0.04)$ and depression ( $\mathrm{p}: 0.04)$.

Conclusion: The results revealed factors significantly associated with poor dyspnea perception despite an obstructive ventilatory disorder. These factors could usefully be considered to successfully manage asthma as well as the regular prescription of an objective test like spirometry.
\end{abstract}

Keywords: Asthma; dyspnea perception; spirometry.

DOI: https://dx.doi.org/10.4314/ahs.v20i3.53

Cite as: Jniene A, Achachi L, El Bakkali M, Herrak L, Rhanim A, Aboudrar S, et al. Factors associated with discrepancies between poor dyspnea perception and abnormal lung function in 65 asthmatic patients. Afri Health Sci. 2020;20(3): 1471-1477. bttps:/ / dx.doi.org/10.4314/abs.v20i3.53

\section{Introduction}

Asthma is a serious global health problem affecting 1 to $18 \%$ of the population with an increasing prevalence in many countries and especially among children. ${ }^{1}$

\section{Corresponding author:}

Asmaa Jniene,

Laboratory of Physiology,

Faculty of Medicine and Pharmacy, impasse souissi, BP: 10100, Rabat, Morocco.

Tel: +212661050099

E-mail: asmaa.jniene@um5.ac.ma
According to the World Health Organization estimates, 235 million people suffer from asthma that occurs in all countries regardless of level of development. ${ }^{2}$ In 2008, a study showed that the prevalence and burden of asthma in te North African countries of Algeria, Morocco and Tunisia was respectively $3.45 \%, 3.89 \%$ and $3.53 \%$ with a high impact on the patients' life. ${ }^{3}$

Asthma is defined by the history of respiratory symptoms, including dyspnea, that vary over time and intensity, together with variable expiratory airflow limitation. The diagnosis and management should be based on the history of characteristic symptom patterns and evidence of variable airflow limitation. ${ }^{1}$ 
The patient's perception of his/her dyspnea in response to change in lung function is nonspecific and assessment directed toward the intensity of dyspnea alone may not accurately reflect the level of persistent functional abnormalities. ${ }^{4}$ Indeed, Dyspnea is a multidimensional and subjective perception of breathing difficulty perceived by the person experiencing it. This symptom can be defined as the subjective experience of breathing discomfort, which comprises qualitatively distinct sensations whose intensity can vary. These sensations originate from interactions among multiple physiological, social, psychological and environmental factors. ${ }^{5,6}$ As there is no single or exclusive stimulus clearly identified inducer of dyspnea, this one is generally evaluated according to a variable whose choice is based on the context. For instance, in a patient with asthma, the level of bronchial obstruction will be considered as a stimulus. ${ }^{7}$ Several studies in recent years have explored the correlation between dyspnea and the degree of airways obstruction in asthmatics patients using measurement tools including both one-dimensional and multidimensional scales that measure changes in the perception of dyspnea. ${ }^{8-18}$ The main finding was a discrepancy between objectively measured lung function and subjectively reported symptoms.

It appears that perception of asthma symptoms is characterized by an interindividual variability. This may lead to inappropriate asthma management and under-treatment thereby placing the patient at risk of severe exacerbations. ${ }^{10,13-15,19}$ Indeed, recent evidence shows that, in real life scenario, asthmatic patients can have poor perception of their symptoms or/and overestimate their level of asthma control, and this can obviously confound the clinical picture and favor sudden worsening of symptoms. ${ }^{20}$

It is of clinical importance to identify patients who are likely to have a poor perception of their symptoms. The determination of some factors associated with poor ability of perceiving bronchial obstruction might be helpful therefore in protecting these patients from a potential severe asthmatic attack. ${ }^{15}$ Several studies were done that documented some factors associated in these patients with a blunted perception of dyspnea but no previous study was done in our context. ${ }^{8,10,21,22}$

The main purpose of our study was to examine the relation between no perceived dyspnea, despite an obstructive ventilatory disorder assessed by spirometry, and other factors in patients with moderate persistent asthma. This will allow to highlight factors which may impact on dyspnea perception in these patients in order to possibly identify such individuals ahead of time for a successful management of the disease.

\section{Material and methods Subjects and inclusion criteria}

Our study was prospective and voluntary, including asthmatic patients who attended their scheduled follow-up pulmonology consultation at Ibn Sina Hospital and recruited between November 2016 and January 2018.

During this follow up consultation, patients routinely receive interrogation, including evaluation of their control according to GINA 2016 (Global Initiative for Asthma), physical examination as well as spirometry over 1 day before their medical appointment. Our investigation took place during these follow-up consultations and included the questionnaire prepared for this study.

\section{Inclusion criteria}

Patients aged more than 18 years

Patients with confirmed moderate persistent asthma followed for more than 1 year. The severity of asthma was determined by the physician according to symptomatic evaluation and the daily medication regimen based on the 2016 guidelines of GINA. Moderate asth$\mathrm{ma}$ is asthma that is well controlled with Step 3 treatment e.g. low dose inhaled corticosteroid/long acting bronchodilators;

Patients with controlled moderate persistent asthma according to 2016 GINA assessment of asthma control (no respiratory symptoms including no dyspnea perception by daytime nor by night), and patients with partly controlled moderate persistent asthma (including only one positive item according to 2016 GINA assessment of asthma control i.e. daytime asthma symptoms represented by dyspnea perception more than twice per week).

Patients with an obstructive ventilatory disorder defined by a FEV1/FVC $<0.7$ and the FEV1 of predicted ranging between 60 and $80 \%$ on the spirometry.

\section{Exclusion criteria}

Patients were excluded if they were on exacerbation or if they presented with any of these conditions: smoking any form of tobacco within 10 years or a history of total cigarette consumption of 10 pack years or more, pregnancy; cognitive impairment; cardiac disorder; current treatment with $B$ adrenergic antagonists or with oral $B$ adrenergic agonists, calcium antagonists, or angiotensin converting enzyme inhibitors; past or present thyroid 
disorder; treatment with systemic corticosteroids in the previous six weeks or respiratory tract infection in the previous six weeks.

\section{Ethical approval}

The Ethical Committee of the Faculty of Medicine and Pharmacy (CERB), Mohammed V University, Rabat, Morocco, approved the study protocol. The notice of information was detailed, and participants consented to take part to this study before their participation.

\section{Measurements}

A structured interview was used to collect data based on a questionnaire that consisted of six parts:

- Demographic data including gender, age, profession, matrimonial status; socio-economic status (The low socio-economic environment was defined with a salary lower than $\$ 200$ per month).

- Asthmatic disease data including antecedents, associated comorbidities including rhinitis, prescribed asthma medication, control and severity of asthma according to GINA 2016.

- HADS (Hospital Anxiety and Depression Scale) for anxiety and depression assessment;

- $\quad$ Perception of dyspnea based on the modified Medical Research Council (mMRC) scale;

- Physical examination data;

- $\quad$ Spirometric data.

The diagnosis of asthma was established in the patients according to 2016 GINA criterion by their symptoms of recurrent episodic wheezing, cough and/or dyspnea, accompanied by the improvement in FEV1 of $\geq 12 \%$ and at least $200 \mathrm{ml}$ after anti-asthma treatment. The obstructive ventilatory disorder was defined by a FEV1/ FVC $<0.7$ (FEV1: forced expiratory volume in 1 second, FVC: Forced Vital Capacity).

FVC and FEV1 were measured by spirometry (Body

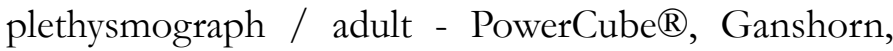
ref: 011400122, software: LF8.5, GLI-2012 equations, which allows us to realize either a spirometry or a plethysmography). Values are expressed as the percentage of the predicted normal value before bronchodilator test.

\section{Psychological status}

Psychological status related to anxiety and depression was evaluated by the HADS. It consists of 14 items, seven for anxiety and seven for depression. Each item is scored from 0 to 3 , where the score of 3 represents a state corresponding to the worst anxiety or depression. The sum of these items produces 2 subscales ranging from 0 to $21 .{ }^{23}$ Each subscore indicates an abnormal case when it is above ${ }^{10}$.

\section{Evaluation and Scoring of dyspnea}

In this study, we define perception of dyspnea as the subjective quantification of the effort stimulus intensity to trigger dyspnea. Self-reported dyspnea symptom ratings as a measure of intensity of dyspnea can be estimated by employing a category ratio scale, such as the modified Medical Research Council (mMRC) scale that is a commonly used validated scale to assess dyspnea in daily living in chronic respiratory diseases including asthma. It is a unidimensional assessment of how dyspnea/breathlessness limits activity and affects functional ability, employment (disability), quality of life, and health status. ${ }^{5}$ This scale evaluates thus dyspnea in daily living and consists of five statements that describe almost the entire range of dyspnea from none (Grade 0 ) or slight without daily life dyspnea perception (Grade 1) to moderate (Grade 2), severe (Grade 3 ) and almost complete incapacity (Grade 4).

The questionnaire was developed in the 1950s by the Medical Research Council with the aim of detecting pneumoconiosis in coal workers, and was later standardized by Fletcher. ${ }^{24}$ Since then, it has been validated in several chronic respiratory diseases, including asthma. ${ }^{25}$ In our study, we compared two groups according to their reported dyspnea perception: those with mMRC $<2$ without daily life dyspnea perception (poor dyspnea perceivers) and those with mMRC scale $\geq 2$ with daily life dyspnea perception.

\section{Statistical analysis}

Statistical Analysis System IBM SPSS Statistics V20.0.0 was used to analyze survey data. Variables were compared between groups with and without daily life dyspnea (as measured by the mMRC scale $\geq 2$ ) despite the presence of an obstructive ventilatory disorder. A chisquare test was used for the univariate analysis of categorical variables while an independent sample t-test/ Mann-Whitney U test was used for continuous data. The threshold of significance was fixed at $\mathrm{p}<0.05$.

\section{Results}

\section{Study population}

Sixty-five patients (39 male) with asthma were enrolled for this study. The main clinical characteristics of the patients are presented in (Table 1). 
Table 1: Baseline sociodemographic and health characteristics of sample $(\mathrm{N}=65)$

\begin{tabular}{|l|l|}
\hline Characteristic & Finding \\
\hline Mean age, years \pm SD & $36 \pm 4.8$ \\
\hline Male sex & $39(60)$ \\
\hline Low socioeconomic income & $25(38)$ \\
\hline Education less than high school & $40(61)$ \\
\hline Associated non treated rhinitis & $41(63)$ \\
\hline Associated gastroesophageal reflux disease & $13(20)$ \\
\hline Onset of symptoms $>10$ years & $49(75)$ \\
\hline Antecedent of near fatal attacks & $4(6)$ \\
\hline $\begin{array}{l}\text { BMI }\left(\text { Kg/ } \text { m }^{2}\right) \\
<25 \\
\geq 25\end{array}$ & $24(37)$ \\
\hline FEV1 $\%$ of predicted \pm SD & $41(63)$ \\
\hline FVC $\%$ of predicted \pm SD & $70 \pm 8$ \\
\hline FEV1/FVC & $91 \pm 8$ \\
\hline mMRC score $<2$ & $0.61 \pm 0.7$ \\
\hline HADS anxiety score $>10$ & $21(32)$ \\
\hline HADS depression score $>10$ & $15(23)$ \\
\hline
\end{tabular}

Data are expressed as number (percentage) or mean \pm standard deviation. FEV1: forced expiratory volume in one second.

FVC: forced vital capacity

BMI: Body mass index

Factors associated with poor dyspnea perception 21 patients $(32.3 \%)$ reported poor dyspnea perception despite an obstructive ventilatory disorder with a FEV1 ranging between 60 and $80 \%$ and a FEV1/FVC $<0.7$. Table 2 shows the comparison between patients with $(\mathrm{mMRC}<2)$ and patients with $\mathrm{mMRC} \geq 2$ regarding baseline sociodemographic and health characteristics.
Based on the univariate analysis (Table 2), it appeared that several factors were statistically associated with poor dyspnea perception: male sex ( $p: 0.03$ ), low socio-economic income ( $p: 0.01$ ), an onset of symptoms greater than 10 years ( $\mathrm{p}: 0.01)$, patients with BMI $\geq 25 \mathrm{Kg} / \mathrm{m} 2$ (p: 0.04) and patients with depression (p : 0.04).

Table 2: Univariate analysis of patients with $\mathrm{mMRC}<2$ and patients with $\mathrm{mMRC} \geq 2$ regarding baseline Sociodemographic and Health Characteristics.

\begin{tabular}{|l|l|l|l|}
\hline Characteristic & $\begin{array}{l}\mathrm{mMRC}<2 \\
\mathrm{~N}=21\end{array}$ & $\begin{array}{l}\mathrm{mMRC} \geq 2 \\
\mathrm{~N}=44\end{array}$ & $\mathrm{p}$ value \\
\hline Mean age, years \pm SD & $34 \pm 3.5$ & $37 \pm 4.7$ & 0.6 \\
\hline Male sex & $17(80.6)$ & $22(50)$ & 0.03 \\
\hline Low socioeconomic income & $10(47.6)$ & $15(34.1)$ & 0.01 \\
\hline Education less than high school & $11(52.4)$ & $29(65.9)$ & 0.9 \\
\hline Associated non treated rhinitis & $15(71.4)$ & $26(59.1)$ & 0.5 \\
\hline Associated gastroesophageal reflux disease & $6(28.6)$ & $7(15.9)$ & 0.8 \\
\hline Onset of symptoms $>10$ years & $19(90.5)$ & $30(68.2)$ & 0.01 \\
\hline Antecedent of near fatal attacks & $1(4.8)$ & $3(6.8)$ & 0.7 \\
\hline $\begin{array}{l}\left.\text { BMI (Kg } \mathrm{m}^{2}\right) \\
<25\end{array}$ & $5(23.8)$ & $19(43.2)$ & 0.04 \\
$\geq 25$ & $16(76.2)$ & $25(56.8)$ & \\
\hline FEV1 \% of predicted \pm SD & $69 \pm 9$ & $72 \pm 7$ & 0.7 \\
\hline FVC (L) of predicted \pm SD & $88 \pm 6$ & $91 \pm 8$ & 0.7 \\
\hline FEV1/FVC & $0.61 \pm 0.7$ & $0.64 \pm 0.6$ & 0.7 \\
\hline HADS depression score $>10$ & $8(38.1)$ & $5(11.4)$ & 0.04 \\
\hline HADS anxiety score $>10$ & $7(33.4)$ & $8(18.2)$ & 0.5 \\
\hline
\end{tabular}

Data are expressed as number (percentage within the subgroups) or mean \pm standard deviation.

FEV1: forced expiratory volume in one second.

FVC: forced vital capacity 


\section{Discussion}

Our data highlighted factors associated with discrepancies between poor dyspnea perception and abnormal lung function represented by an obstructive ventilatory disorder as assessed by spirometry in 65 patients with moderate persistent asthma. Associated factors were male sex ( $p: 0.03$ ), low socio-economic income ( $p: 0.01$ ), an onset of symptoms greater than 10 years (p: 0.01), patients with BMI $\geq 25$ ( $p: 0.04$ ) and patients with depression ( $p: 0.04$ ).

Dyspnea perception has been previously studied in asthma and inadequate symptom perception has been reported in a variety of previous studies using different methods. ${ }^{10-18}$ In the present study, we assessed dyspnea perception by the mMRC scale. The perception of dyspnea involves a complex chain of events that depend on varying cortical integration of several afferent/efferent signals and coloured by affective processing. ${ }^{26}$ Therefore, assessment of dyspnea depends mainly on self-report. 5 based on validated scales in pulmonary chronic diseases such as the mMRC scale.

The main finding of the previous studies was a discrepancy between objectively measured lung function and subjectively reported symptoms for both over and under-perception of dyspnea. ${ }^{10-18}$ In this context, studies showed that an alteration in the ability of asthmatic patients to perceive symptoms in their day-to-day life, mainly an under-perception of dyspnea, will influence negatively in the management of asthma due to an underestimation of the symptoms severity. ${ }^{13-15}$ Indeed, the delay in instituting treatment is the single most important factor contributing to death from asthma. Particularly in the case of life threatening and fatal asthma attacks, a blunted perception of dyspnea has been assumed to be a causal factor. ${ }^{14-17,27}$ In this regard, Kikuchi et al. experimentally induced dyspnea in patients who had or had not previously experienced near fatal asthma attacks and in healthy volunteers. ${ }^{14}$ Patients with a history of near fatal attacks perceived significantly less dyspnea than patients without such a history and healthy controls. Similarly, in a study by Magadle et al., $26 \%$ of 113 patients with asthma showed blunted symptom perception. During the following 2 years this group, when compared to those patients with accurate symptom perception, exhibited a fourfold rate of visits to the emergency department, a more than five-fold hospitalization rate and a six-fold rate of near fatal and fatal asthma attack. ${ }^{16}$ All of these findings pointed to perception as being a key factor in the overall management of asthma. ${ }^{28}$
On the other hand, several studies reported some factors to be associated with blunted dyspnea perception such as advanced age, the association with the presence of bronchial eosinophilic inflammation, severe asthma and depression status. $8,10,13,21,22,29$

Conolly and al describes reduced awareness of bronchoconstriction induced by methacholine in elderly (asthmatic patients and normal individuals). This impaired perception of bronchoconstriction was applied equally to the asthmatic and the normal subjects suggesting that it may be a feature of aging rather than of asthma but also it may delay self-referral in acute asthma and contribute to higher asthma mortality in the elderly. ${ }^{13,29}$ Among the explanations for the impact of age on dyspnea perception is that subjects become cortically less sensitive to the peripheral signals of dyspnea with increasing age. Also, familiarity with this symptom may lead to desensitization. ${ }^{13,29}$ In our study the age wasn't associated with poor dyspnea perception (most of the patients were aged under 40 years). Otherwise, the duration of the disease beyond 10 years was associated with poor dyspnea perception which can be explained by desensitization due to familiarity with this symptom. This explanation may be also evoked in poor dyspnea perception in overweight subjects. Indeed, while patients with mMRC $<2$ and BMI greater than $25 \mathrm{Kg} /$ $\mathrm{m}^{2}$ were questioned about dyspnea that may accompany overweight, they answered that they were used to it leading to its under-perception.

It is well known that intentional poor therapeutic compliance due to cost, cultural issues and concerns about side-effects are factors contributing to this poor condition in asthma. ${ }^{1}$ Our study showed low socio-economic income was statistically associated to poor dyspnea perception (p: 0.01).

The progression of chronic diseases such as asthma is often associated with a progression of depression status. Affective states can profoundly impact upon the perception of dyspnea for both over and under-perception of dyspnea. Indeed, depression and anxiety as well can increase the intensity of dyspnea out of proportion to the impairment in cardiorespiratory function including asthma and may contributto the degree of disability associated with dyspnea. ${ }^{30}$ A study showed that anxiety but not depression was associated with greater perceived dyspnea intensity after methacholine challenge. ${ }^{31}$ in this regard, our results showed that depression was statistically associated to poor dyspnea perception (p: 0.04) as assessed by HAD scale score for depression 
greater than ${ }^{10}$. In general, whether depression is associated with over or under-perception of dyspnea, psychological states may influence asthma control and indicate that physicians should consider and evaluate the different psychological state of the patient as well as lung function before establishing a treatment regimen $.8,31$

In most studies, it is admitted that dyspnea perception was found to be higher in healthy subjects than in asthmatics and it appears to be essentially limited to severe asthma in several studies. ${ }^{8,10}$ Moreover, the perception of airway narrowing seems to be reduced in subjects with severe asthma and to be associated with an increased risk of attack/exacerbation. in our study, we found a blunted perception of dyspnea in patients with moderate persistent asthma which emphasize to consider this condition as well in this level of severity. In severe asthma, inflammatory activity within the airways of asthmatic subjects could explain differences in dyspnea perception. ${ }^{8}$ In particularly, the eosinophilia in sputum and bronchial biopsies seems to affect dyspnea perception in severe asthmatics and has been suggested as an indicator of clinical instability. ${ }^{8}$ Another study showed also that patients with severe asthma, particularly those with recurrent exacerbations, have blunted perception of dyspnea, which is related to the degree of sputum eosinophilia. ${ }^{22}$ This suggests also that increased sputum eosinophilia is an indicator of clinical instability, and that eosinophilic airways inflammation might affect dyspnea perception in severe asthma. ${ }^{8}$ ${ }^{22}$ Consequently markers of inflammation more than hyperresponsiveness testing to different agonists are needed to give more insight into this issue and could be of clinical importance to identify patients who are likely to have a poor perception of symptoms and could help in advanced practice roles to successfully manage these patients. $^{8}$

There are important clinical messages from these observations: Impaired perception in some patients with moderate persistent asthma with some significant statistically associated factors may conduct to underestimation of the non-control and the severity of the disease. This suggests in a general way, that objective measurement of airflow obstruction by spirometry or peak expiratory flow rate should be encouraged in patients and should be mandatory in any patient known or suspected with low or no perception of dyspnea.

The main limitations of our study are represented by the small sample and the assessment of dyspnea by a subjective measurement that is a scale. Also, the identifiable variables may not be independently associated with the main outcome as the statistical analysis didn't include the multivariate analysis due to the small sample. A further limitation is the difficultly in establishing causality. Longitudinal studies are thus required to clarify the cause-and-effect relationships including a larger sample, as well as objective measurements as an induced bronchoconstriction by methacholine, other testing as the eosinophilia in sputum and bronchial biopsies and also a follow-up of the patients.

\section{Conclusion}

Our study showed factors associated with poor dyspnea perception despite an obstructive ventilatory disorder assessed by spirometry. These factors could usefully be considered when attempting to successfully manage asthma. On the other hand, our findings suggest that questionnaire-based evaluations of symptoms only may not necessarily be reliable. In this context, the prescription of an objective test as spirometry should be regularly prescribed.

\section{Conflict of interest}

None declared.

\section{References}

1. Global strategy for asthma management and prevention. Global Initiative for Asthma. www.ginasthma.org. 2018.

2. https://www.who.int/respiratory/asthma/en/.

3. Nafti S, Taright S, El Ftouh M, Yassine N, Benkheder A, Bouacha H, et al. Prevalence of asthma in North Africa: the Asthma Insights and Reality in the Maghreb (AIRMAG) study. Respir Med. 2009;103 Suppl 2:S2-11.

4. Laveneziana P, Lotti P, Coli C, Binazzi B, Chiti L, Stendardi L, et al. Mechanisms of dyspnoea and its language in patients with asthma. The European Respiratory Journal. 2006;27(4):742-7.

5. Parshall MB, Schwartzstein RM, Adams L, Banzett RB, Manning HL, Bourbeau J, et al. An official American Thoracic Society statement: update on the mechanisms, assessment, and management of dyspnea. American Journal of Respiratory and Critical Care Medicine. 2012;185(4):435-52.

6. Dyspnea. Mechanisms, assessment, and management: a consensus statement. American Thoracic Society. American Journal of Respiratory and Critical Care Medicine. 1999;159(1):321-40.

7. Manning HL, Mahler DA. Pathophysiology of dyspnea. Monaldi archives for chest disease $=$ Archivio Monaldi per le malattie del torace. 2001;56(4):325-30.

8. Foschino Barbaro MP, Lacedonia D, Palladino GP, 
Bergantino L, Ruggeri C, Martinelli D, et al. Dyspnea perception in asthma: Role of airways inflammation, age and emotional status. Respiratory Medicine. 2011;105(2):195-203.

9. Spector N, Klein D. Chronic critically ill dyspneic patients: mechanisms and clinical measurement. $A A C N$ Clinical Issues. 2001;12(2):220-33.

10. Bijl-Hofland ID, Cloosterman SG, Folgering HT, Akkermans RP, van Schayck CP. Relation of the perception of airway obstruction to the severity of asthma. Thorax. 1999;54(1):15-9.

11. Hardie GE, Gold WM, Janson S, Carrieri-Kohlman V, Boushey HA. Understanding how asthmatics perceive symptom distress during a methacholine challenge. The Journal of Asthma: official Journal of the Association for the Care of Asthma. 2002;39(7):611-8.

12. Tetzlaff K, Leplow B, ten Thoren C, Dahme B. Perception of dyspnea during histamine- and methacholine-induced bronchoconstriction. Respiration; international Review of Thoracic Diseases. 1999;66(5):427-33.

13. Barnes PJ. Poorly perceived asthma. Thorax. 1992;47(6):408-9.

14. Kikuchi Y, Okabe S, Tamura G, Hida W, Homma $\mathrm{M}$, Shirato $\mathrm{K}$, et al. Chemosensitivity and perception of dyspnea in patients with a history of near-fatal asthma. The New England Journal of Medicine. 1994;330(19):132934.

15. Barnes PJ. Blunted perception and death from asthma. The New England Journal of Medicine. 1994;330(19):1383-4.

16. Magadle R, Berar-Yanay N, Weiner P. The risk of hospitalization and near-fatal and fatal asthma in relation to the perception of dyspnea. Chest. 2002;121(2):32933.

17. Eckert DJ, Catcheside PG, McEvoy RD. Blunted sensation of dyspnoea and near fatal asthma. The European Respiratory Journal. 2004;24(2):197-9.

18. Barreiro E, Gea J, Sanjuas C, Marcos R, Broquetas J, Milic-Emili J. Dyspnoea at rest and at the end of different exercises in patients with near-fatal asthma. The European Respiratory Journal. 2004;24(2):219-25.

19. Boulet LP, Leblanc P, Turcotte H. Perception scoring of induced bronchoconstriction as an index of awareness of asthma symptoms. Chest. 1994;105(5):1430-3.
20. Patella V, Santus P, Puggioni F, Steinhilber G, Scichilone N. Is mild asthma in real life always in the Green Zone? Minerva Medica. 2014;105(3 Suppl 2):1-6.

21. Roisman GL, Peiffer C, Lacronique JG, Le Cae A, Dusser DJ. Perception of bronchial obstruction in asthmatic patients. Relationship with bronchial eosinophilic inflammation and epithelial damage and effect of corticosteroid treatment. The Journal of Clinical Investigation. 1995;96(1):12-21.

22. Veen JC, Smits HH, Ravensberg AJ, Hiemstra PS, Sterk PJ, Bel EH. Impaired perception of dyspnea in patients with severe asthma. Relation to sputum eosinophils. American Journal of Respiratory and Critical Care Medicine. 1998;158(4):1134-41.

23. Zigmond AS, Snaith RP. The hospital anxiety and depression scale. Acta Psychiatrica Scandinavica. 1983;67(6):361-70.

24. Fletcher CM. The clinical diagnosis of pulmonary emphysema; an experimental study. Proceedings of the Royal Society of Medicine. 1952;45(9):577-84.

25. Mahler DA, Wells CK. Evaluation of clinical methods for rating dyspnea. Chest. 1988;93(3):580-6.

26. Laviolette L, Laveneziana P. Dyspnoea: a multidimensional and multidisciplinary approach. The European Respiratory Journal.. 2014;43(6):1750-62.

27. von Leupoldt A, Dahme B. Psychological aspects in the perception of dyspnea in obstructive pulmonary diseases. Respir Med. 2007;101(3):411-22.

28. Nannini LJ, Zaietta GA, Guerrera AJ, Varela JA, Fernandez OM, Flores DM. Breath-holding test in subjects with near-fatal asthma. A new index for dyspnea perception. Respir Med. 2007;101(2):246-53.

29. Connolly MJ, Crowley JJ, Charan NB, Nielson CP, Vestal RE. Reduced subjective awareness of bronchoconstriction provoked by methacholine in elderly asthmatic and normal subjects as measured on a simple awareness scale. Thorax. 1992;47(6):410-3.

30. Scano G, Gigliotti F, Stendardi L, Gagliardi E. Dyspnea and emotional states in health and disease. Respiratory Medicine. 2013;107(5):649-55.

31. Li HL, He XL, Liang BM, Zhang HP, Wang Y, Wang G. Anxiety but not depression symptoms are associated with greater perceived dyspnea in asthma during bronchoconstriction. Allergy and Asthma Proceedings. 2015;36(6):447-57. 